\title{
Signature of biased range in the non-dynamical Chern-Simons modified gravity and its measurements with satellite-satellite tracking missions: theoretical studies
}

\author{
Li-E Qiang ${ }^{1, \mathrm{a}}$, Peng $\mathrm{Xu}^{2, \mathrm{~b}}$ (]) \\ ${ }^{1}$ Department of Geophysics, College of Geology Engineering and Geomatics, Chang'an University, Xi' an 710054, China \\ ${ }^{2}$ Academy of Mathematics and Systems Science, Chinese Academy of Sciences, Beijing 100190, China
}

Received: 9 May 2014 / Accepted: 11 August 2015 / Published online: 26 August 2015

(C) The Author(s) 2015. This article is published with open access at Springerlink.com

\begin{abstract}
Having great accuracy in the range and range rate measurements, the GRACE mission and the planed GRACE follow on mission can in principle be employed to place strong constraints on certain relativistic gravitational theories. In this paper, we work out the range observable of the non-dynamical Chern-Simons modified gravity for the satellite-to-satellite tracking (SST) measurements. We find out that a characteristic time accumulating range signal appears in non-dynamical Chern-Simons gravity, which has no analogue found in the standard parity-preserving metric theories of gravity. The magnitude of this Chern-Simons range signal will reach a few times of $\chi \mathrm{cm}$ for each free flight of these SST missions, here $\chi$ is the dimensionless postNewtonian parameter of the non-dynamical Chern-Simons theory. Therefore, with the 12 years data of the GRACE mission, one expects that the mass scale $M_{C S}=\frac{4 \hbar c}{x a}$ of the non-dynamical Chern-Simons gravity could be constrained to be larger than $1.9 \times 10^{-9} \mathrm{eV}$. For the GRACE FO mission that scheduled to be launched in 2017, the much stronger bound that $M_{C S} \geq 5 \times 10^{-7} \mathrm{eV}$ is expected.
\end{abstract}

\section{Introduction and motivations}

Einstein's general theory of relativity (GR), as the fundamental theory for gravitation and dynamical spacetime, is one of the corner stones of modern physics and cosmology. From the late 1960s, with the establishment of the Dicke framework [1] and the parameterized post-Newtonian (PN) formalism [2-5], GR had passed many stringent tests with scales ranging from $1 \mathrm{~mm}$ to $1 \mathrm{kAU}[6,7]$. While, recently, observations from astrophysics and cosmology had given rise to new chal-

\footnotetext{
a e-mail: qqllee815@chd.edu.cn

b e-mail: xupeng@amss.ac.cn
}

lenges to GR, which are known as the dark matter and dark energy problems [8-12]. Concerning these, different classes of modified gravitational theories had been developed, please consult [13] for details. On the other hand, in searching for the union of quantum mechanics and gravity, modifications and extensions to GR also arose naturally along the different approaches to this "Holy-Grail" of fundamental physics.

Among the modified theories, the extensions to the Einstein-Hilbert action with second order curvature terms are of particular interest, which may arise in the full, but still lacking, quantum theory of gravity as high energy corrections to GR, see [14]. The string theory inspired ChernSimons (CS) modified gravity [15-19], with the additions of a parity-violating term $R{ }^{\star} R$ and a coupling scalar field $\theta$, is one of such extensions. CS modified gravity appeared first in [15] as a $(2+1)$-dimensional model, and then it was extended into $3+1$ dimensions as a consequence of the string theory $[16,17]$. Now, it is known that CS gravity is required by all 4-dimensional compactifications of string theory for self-consistency [20]. Being a promising model, CS modified gravity has found connections with different fields such as gravitational physics, particle physics, string theory, loop quantum gravity, and cosmology, please consult [19] for detailed discussions.

CS modified gravity now contains two classes of formulations, the non-dynamical and dynamical formulations, which are in fact two distinct theories. In the non-dynamical formulation, the CS scalar $\theta$ is externally prescribed, which depends on the mass scale $M_{C S}$ of the specific theory under the consideration. while, in the more realistic but complicate dynamical formulation, the evolution of the CS scalar is then sourced by the spacetime curvature. The non-dynamical CS gravity now serves as a useful model that provides us insights into parity violating theories of gravity. Up to now, the tests and constraints on both the non-dynamical and dynamical CS 
gravity are all based on the observations from astrophysics and space based experiments. The first but weak bound on the CS scalar $\theta$ or the mass scale was obtained in [21] based on the results from the LAGEOS I, II [22-24] and the Gravity Probe-B [25] missions, which had placed the constraint $M_{C S} \geq 2 \times 10^{-13} \mathrm{eV}$. In the attempts to explain the flatness of galaxy rotation curves, new bound was also obtained [26]. The strongest bound on the CS mass scale up to now was based on the data from double binary pulsars [27,28], which had the constraint $M_{C S} \geq 4.7 \times 10^{-10} \mathrm{eV}$ as been revised in [28]. For the tests of the dynamical CS gravity, the studies became active only recently. The vacuum solutions outside rotating black holes and stars in the dynamical formulation were studied with the slow rotation approximation [29-31], and their possible tests can be found in [32-34]. Moreover, the parity-violating term $R{ }^{\star} R$ also leaves distinguishable signatures in gravitational waves, which may be captured by ground based or future space borne gravitational wave antennas [35-38].

Based on the $\mathrm{PN}$ analysis of the non-dynamical CS gravity in $[39,40]$, we suggest here a new method to place a rather strong constraint on the mass scale of the non-dynamical theory. A characteristic range observable $\delta \rho^{C S}$ is found here for the non-dynamical theory which could be measured by the operating and future planned satellite-to-satellite tracking (SST) missions, that the GRACE and GRACE Follow On (GRACE FO) missions. To summarize here

$\delta \rho^{C S}=-\frac{\chi G J \rho_{0} \sin i}{2 c^{2} a^{3}}\left(\sin (\omega t) t+\frac{\cos (\omega t)}{\omega}\right)$,

where $\chi$ is the new PN parameter of the non-dynamical theory [39], and $J$ denotes the Earth angular momentum. $i, \omega$, and $a$ denote the inclination, angular frequency and semi-major of the orbit of the GRACE or GRACE FO satellites, and $\rho_{0}$ denotes the averaged range between the two satellites. The key result turns out to be that the CS range observable contains an oscillating term that growing linearly with time, which has no analogue found in standard paritypreserving metric theories. In each free flight of these SST missions, $\delta \rho^{C S}$ will reach to a few $\chi \mathrm{cm}$. With the 12 years data from the GRACE mission, one expects that the mass scale of the non-dynamical CS gravity will be constrained to $M_{C S} \geq 1.9 \times 10^{-9} \mathrm{eV}$. For the future GRACE FO mission, an even stronger bound, that $M_{C S} \geq 5 \times 10^{-7} \mathrm{eV}$, is expected. Therefore, in principle, with the help of these SST missions, one could place, up to now, the strongest constraints on the CS modified gravity.

This paper focuses on the theoretical studies and expands as follows. We first give a brief introductions on the status of the GRACE and GRACE FO missions in Sect. 2. The nondynamical CS modified gravity is briefly reviewed in Sect. 3 . The detailed derivations of our results is described in Sect. 4 .
Finally, we discuss the measurements of the CS range signal with the GRACE and GRACE FO missions in Sect. 5. As mentioned before the non-dynamical CS gravity can only serves as a model mimic the the more realistic dynamical one. The studies of the range observable in dynamical CS gravity in the slow rotation approximation will be left in future works.

\section{GRACE and GRACE follow on missions}

The Gravity Recovery And Climate Experiment (GRACE) mission is a joint mission between the National Aeronautics and Space Administration (NASA) in the United States and the Deutsche Forschungsanstalt für Luft und Raumfahrt in Germany, which was launched in March of 2002 [41]. The aim of GRACE mission is to accurately map the variations of Earth gravity field for a nominal mission lifetime of five years. Today, GRACE is still operating in an extended mission phase, which is expected to continue through at least $2015 .{ }^{1}$ GRACE is a SST mission at low Earth orbit, which is consisted of two identical satellites that following almost the same near circular polar orbit one after another, please see Table 1 for the samples of the orbit elements.

The two satellites are separated along-track from each other by $170-270 \mathrm{~km}$ maintained by occasional orbit maintenance manoeuvres, and linked continuously by highly accurate inter-satellite K-Band Ranging system. The SST measurement has the accuracy about $10 \mu \mathrm{m} / \sqrt{\mathrm{Hz}}$ for biased range and about $1 \mu \mathrm{m} / \mathrm{s} \sqrt{\mathrm{Hz}}$ for range rate in the signal band of $10^{-2}-10^{-1} \mathrm{~Hz}[41,42]$. Near the orbital frequency $\sim 10^{-4} \mathrm{~Hz}$, the accuracy in the range measurement is still about 1-2 cm [42]. To be brief, such high accuracy is obtained by the combination of the dual-frequency one-way K-Band phase measurements carried on each satellite, which can largely remove the noises from the instability of the on-board ultra stable oscillators and errors from Earth ionosphere. Also, GRACE carries accelerometers to remove effects from non-gravitational forces and Global Positioning Systems to provide both the precise time-tags for the recorded data and the positions of the satellites over Earth.

To continue the critical Earth gravity variation data recorded by GRACE, NASA has scheduled the launch of the GRACE Follow On mission to August 2017. The GRACE FO mission would re-fly the identical GRACE spacecraft and instruments, but supplement the micrometre-level accuracy microwave measurement with a laser interferometer of nanometre-level accuracy. ${ }^{2}$ From the detailed simulations

\footnotetext{
1 Please see http://www.csr.utexas.edu/grace/, http://www.nasa.gov/ mission_pages/Grace/index.html and https://earth.esa.int/web/guest/ missions/3rd-party-missions/current-missions/grace.

${ }^{2}$ Please see https://www.aei.mpg.de/18528/04_Grace_Follow-on.
} 
Table 1 Samples of the GRACE orbit elements, which are the semimajor axis $a$, the eccentricity $e$, the orbital inclination $i$ to the Earth equator, the longitude of the ascending node $\Omega$, the argument of peri- center $\omega$, and the mean anomaly $\mathscr{M}$. The Keplerian orbital periods of the GRACE pair are of the order of $1.56 \mathrm{~h}$. This data is from 13 September 2003

\begin{tabular}{lllllll}
\hline S/C & $a(\mathrm{~km})$ & $e$ & $i\left(^{\circ}\right)$ & $\Omega\left(^{\circ}\right)$ & $\omega\left(^{\circ}\right)$ & $\mathscr{M}\left({ }^{\circ}\right)$ \\
\hline GRACE A & 6841.11877 & 0.00272831 & 89.9395 & -71.5742 & 119.916 & -179.997 \\
GRACE B & 6839.80210 & 0.00298412 & 89.8374 & -71.5081 & 118.082 & -179.997 \\
\hline
\end{tabular}

of the laser ranging system of GRACE FO [43-45], in the signal band $10^{-2}-10^{-1} \mathrm{~Hz}$, the range measurements accuracy is about $100 \mathrm{~nm} / \sqrt{\mathrm{Hz}}$ for $270 \mathrm{~km}$ satellites separation and $1 \mathrm{pm} / \sqrt{\mathrm{Hz}}-0.1 \mathrm{~nm} / \sqrt{\mathrm{Hz}}$ for $50 \mathrm{~km}$ separation. Near the orbital frequency $\sim 10^{-4} \mathrm{~Hz}$, the accuracy is still about $200 \mu \mathrm{m} / \sqrt{\mathrm{Hz}}$ for $270 \mathrm{~km}$ satellites separation and $10 \mu \mathrm{m} / \sqrt{\mathrm{Hz}}$ for $50 \mathrm{~km}$ separation.

The GRACE and GRACE FO missions, in the first place, are not designed for the tests of relativistic theories of gravitation. While, as discussed above, the great accuracy in the range and range rate measurements, especially for the case of the laser ranging system of GRACE FO, are in principle possible to place strong constraints on certain relativistic gravitational theories, please also consult the works [46-48] along this line.

\section{Non-dynamical Chern-Simons modified gravity}

We give a brief introduction to the non-dynamical formulation of CS modified gravity, for detailed discussions please consult $[19,39,40]$. The geometric units $c=G=1$ are adopted. The action for the non-dynamical CS gravity reads

$S:=S_{G R}+S_{C S}+S_{\text {matt }}$,

where

$S_{G R}=\frac{1}{16 \pi} \int d^{4} x \sqrt{-g} R$

$S_{C S}=\frac{\alpha}{4} \int d^{4} x \sqrt{-g} \theta R^{\star} R$

and $S_{\text {matt }}$ is the action from the matter fields which is independent of $\theta . g$ is the determinant of the metric and the Pontryagin density reads

$R^{\star} R=\frac{1}{2} \epsilon^{c d e f} R_{b e f}^{a} R_{a c d}^{b}$

The magnitude of the CS extension is controlled by the coupling field $\theta$, which is externally prescribed and depends on the mass scale of the specific theory that under the consideration. $\theta$ can also be viewed as the deformation function, and the difference between CS gravity and GR is proportional to the deformation parameters $\nabla_{a} \theta$ and $\nabla_{a} \nabla_{b} \theta$. In this work, the most popular choice, called the canonical coupling
[18], is adopted, where $\theta$ is a spatially homogeneous function and depends linearly on time. Therefore the deformation parameter contains only $\dot{\theta}$. With such choice, spacetimedependent reparameterization of spacial variables and time translation remain symmetries of the CS modified theory $[19,39]$.

The field equation of the non-dynamical CS gravity is obtained by varying the action with respect to the metric

$R_{a b}-\frac{1}{2} g_{a b} R+16 \pi \alpha C_{a b}=8 \pi T_{a b}$,

where $C_{a b}$ is the 4-dimensional generalization of the CottonYork tensor

$C^{a b}=\nabla_{c} \theta \epsilon^{c d e(a} \nabla_{e} R_{d}^{b)}+\frac{1}{2} \nabla_{c} \nabla_{d} \theta \epsilon^{e f d(a} R_{f e}^{b) c}$

The introduction of the new scalar degree of freedom $\theta$ also gives rise to the new constraint

$\nabla_{a} C^{a b}=-\frac{1}{8} \nabla^{b} \theta\left({ }^{\star} R R\right)=0$.

If the above constraint is satisfied, from Eq. (4), the Bianchi identities and the equations of motion for matter fields $\nabla_{a} T^{a b}=0$ are recovered, which rank the nondynamical CS gravity a metric theory.

In the weak field and slow motion limits, the parametrized post-Newtonian (PPN) metric of the non-dynamical CS gravity outside a compact source was carefully worked out in $[39,40]$. As mentioned before, the non-dynamical CS gravity differs from GR only in the gravitomagnetic sector

$g_{0 i}^{C S}=g_{0 i}^{G R}+\chi(r \nabla \times \mathbf{V})_{i}$,

here $r$ denotes the distance to the mass center of the compact source and $V_{i}$ is the PN potential, see Appendix A. The dimensionless parameter $\chi=\frac{32 \pi \alpha \dot{\theta}}{r}$ is the new PN parameter for non-dynamical CS gravity, and the CS mass scale reads $[39,40]$

$M c s=\frac{1}{8 \pi \alpha \dot{\theta}}=\frac{4}{\chi r}$. 


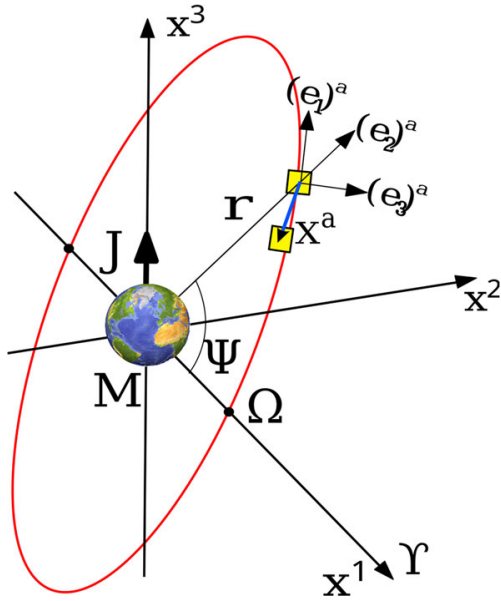

Fig. 1 The settings of the PN reference frame and the local frame of the reference satellite. The orbits under consideration are circular orbits with the longitude of ascending node $\Omega=0$. As illustrated in the figure, the local tetrad carried by the reference satellite is defined as follows, $\left(\mathbf{e}_{1}\right)^{a}$ is set along the direction of motion of the reference satellite, $\left(\mathbf{e}_{2}\right)^{a}$ along the radial direction and $\left(\mathbf{e}_{3}\right)^{a}=\left(\mathbf{e}_{1}\right)^{a} \times\left(\mathbf{e}_{2}\right)^{a}$

\section{The range observable of non-dynamical Chern-Simons gravity}

\subsection{The basic settings}

According to the SST missions introduced in Sect. 2, we study the range observable between the two satellites, which are modeled here as two proof masses orbiting Earth one after another along nearly circular orbits. We restrict ourselves to the so-called "semi-conservative" metric theories, which are based on action principles and respect the conservation law of 4-momentum [7]. Therefore, the relevant $\mathrm{PN}$ parameters are $\left\{\gamma, \beta, \xi, \alpha_{1}, \alpha_{2}\right\}$ together with the additional CS parameter
Such coordinate directions are tied to the remote stars, and the time $t$ is measured by the observers at asymptotically flat region. Within our coordinate system the PN metric outside Earth reads

$$
\begin{aligned}
g_{00}= & -1+2 U-2 \beta U^{2}-2 \xi \Phi_{W}+(2 \gamma+2-2 \xi) \Phi_{1} \\
& +2(3 \gamma-2 \beta+1+\xi) \Phi_{2}+2 \Phi_{3}+2(3 \gamma-2 \xi) \Phi_{4} \\
& +2 \xi \mathscr{A}-\left(\alpha_{1}-\alpha_{2}\right) w^{2} U-\alpha_{2} w^{i} w^{j} U_{i j} \\
& -2 \alpha_{1} w^{i} V_{i}+\mathscr{O}\left(\epsilon^{6}\right), \\
g_{0 i}= & -\frac{1}{2}\left(4 \gamma+3+\alpha_{1}-\alpha_{2}-2 \xi\right) V_{i}-\frac{1}{2}\left(1+\alpha_{2}+2 \xi\right) W_{i} \\
& +\chi r(\nabla \times \mathbf{V})_{i}-\frac{1}{2}\left(\alpha_{1}-2 \alpha_{2}\right) w_{i} U \\
& -\alpha_{2} w^{j} U_{i j}+\mathscr{O}\left(\epsilon^{5}\right), \\
g_{i j}= & (1+2 \gamma U) \delta_{i j}+\mathscr{O}\left(\epsilon^{4}\right),
\end{aligned}
$$

please see Appendix A for the PN potentials. For low and medium Earth orbits experiments, the magnitude of $\epsilon$ is about $10^{-5}$.

We model Earth as an ideal and uniform rotating spherical body. The preferred-frame and the preferred-location effects are tightly constrained by observations, and we now have the upper bounds of the related PN parameters as $\alpha_{1} \sim 4 \times 10^{-5}, \alpha_{2} \sim 2 \times 10^{-9}, \alpha_{3} \sim 4 \times 10^{-20}$ and $\xi \sim 10^{-9}$, please see Table 2 or [7] for more details. Generally, the coordinate velocity $w$ of the PPN coordinate system relative to the mean rest-frame of the universe is believed to be small, that $w \sim \mathscr{O}(\epsilon)[4,7,49]$. Therefore, the gradients produced by the preferred-frame and the preferred-location effects between the two orbiting satellites will be smaller than $10^{-21} \mathrm{~s}^{-2}$, which will produce a relative acceleration smaller than $2 \times 10^{-16} \mathrm{~m} / \mathrm{s}^{2}$. This is too small to be seen by the present day and future planned SST missions and will be ignored in this work. The above metric can then be cast into a rather simple form

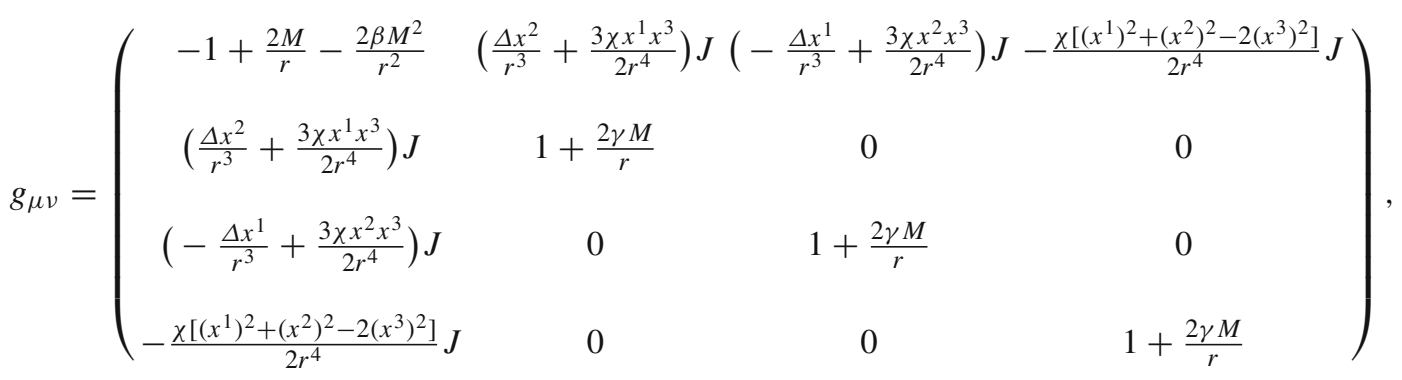

$\chi$, please see [7] or Appendix A for the parametrized PN formalism. The PN coordinates system $\left\{t, x^{i}\right\}$ outside Earth is chosen as follows. The mass center of Earth is set at the origin. The basis $\left(\frac{\partial}{\partial x^{3}}\right)^{a}$ is set to parallel to the direction of the Earth angular momentum $\mathbf{J},\left(\frac{\partial}{\partial x^{1}}\right)^{a}$ is pointing to a reference star $\Upsilon$ and $\left(\frac{\partial}{\partial x^{2}}\right)^{a}$ determined by the right-hand rule $\left(\frac{\partial}{\partial x^{1}}\right)^{a} \times\left(\frac{\partial}{\partial x^{2}}\right)^{a}=\left(\frac{\partial}{\partial x^{3}}\right)^{a}$, see Fig. 1 for illustration. where $r=\sqrt{\delta_{i j} x^{i} x^{j}}, \Delta=1+\gamma+\frac{1}{4} \alpha_{1}$, and $M, \mathbf{J}$ are the asymptotically measured total mass and angular momentum of Earth

$$
\begin{aligned}
M & =\int \rho\left[1+(\gamma+1) v^{2}+(3 \gamma-2 \beta+1) U+\frac{\Pi}{\rho}+3 \gamma \frac{p}{\rho}\right] d^{3} x, \\
\mathbf{J} & =\int \rho(\mathbf{x} \times \mathbf{v}) d^{3} x .
\end{aligned}
$$


Table 2 Current values of PPN parameters

\begin{tabular}{lll}
\hline Parameter & Bound & Experiment \\
\hline$\gamma-1$ & $2.3 \times 10^{-5}$ & Time delay in Cassini tracking \\
$\beta-1$ & $2 \times 10^{-4}$ & Light deflection in VLBI \\
& $8 \times 10^{-5}$ & Perihelion shift \\
$\xi$ & $2.3 \times 10^{-4}$ & Nordtvedt effect \\
$\xi$ & $10^{-9}$ & Spin precession of millisecond pulsars \\
$\alpha_{1}$ & $4 \times 10^{-5}$ & Orbital polarization of PSR J1738 +0333 \\
& $10^{-4}$ & Lunar laser ranging \\
$\alpha_{2}$ & $2 \times 10^{-9}$ & Spin precession of millisecond pulsars \\
$\alpha_{3}$ & $4 \times 10^{-20}$ & Pulsar spin down statistics \\
$\zeta_{1}$ & 0.02 & Combined PPN bounds \\
$\zeta_{2}$ & $4 \times 10^{-5}$ & Binary acceleration of PSR 1913 + 16 \\
$\zeta_{3}$ & $10^{-8}$ & Lunar acceleration \\
$\zeta_{4}$ & - & Not independent \\
\hline
\end{tabular}

For a satellite orbiting Earth with velocity $v$, one has the basic order relations

$v^{2} \sim \frac{M}{r} \sim \mathscr{O}\left(\epsilon^{2}\right), \quad v^{4} \sim \frac{M^{2}}{r^{2}} \sim \frac{J v}{r^{2}} \sim \mathscr{O}\left(\epsilon^{4}\right)$.

One should notice that the leading gradients acting on the two satellites are of the Newtonian ones, the PN gradients are generally of $\mathscr{O}\left(\epsilon^{2}\right) \sim 10^{-10}$ times smaller than the Newtonian gradients. At the PN level, the deviations of the centered matter source from ideal uniform sphere will give rise to multipolar corrections to the Newtonian potential in the $g_{00}$ component

$U=\frac{M}{r}+\frac{M}{r} \sum_{l=2}^{\infty} \frac{R^{l}}{r^{l}} \sum_{m=-l}^{l} C_{l m} Y_{l m}$

where $R$ is the mean radius of Earth and $C_{l m}$ is the coefficient of the spherical harmonic component. Potentials from these multiples belongs to purely non-relativistic effects, and along low Earth orbits they are generally smaller than $\mathscr{O}\left(\epsilon^{3}\right)$ with the only exception of the $J_{2} \sim 10^{-3} \frac{R^{2}}{r^{2}} \frac{M}{r}$ component. ${ }^{3} \mathrm{We}$ ignore such multiples in the following theoretical analysis in this section. The effects of these multiples on the measurements and the possible data analysis methods will be briefly discussed in Sect. 5.

\subsection{The geodesic deviation equation}

The first step studies of the geodesic motions of proof masses in CS modified gravity and their orbital observable can be found in $[21,32]$. While, in this work, since we are interested in the range observable between the two satellites instead,

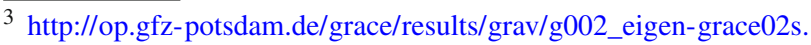
html.
}

the geodesic deviation equation that describes their relative motions will be a proper starting point

$T^{b} \nabla_{b} T^{c} \nabla_{c} X^{a}+R_{b c d}^{a} T^{b} T^{d} X^{c}=0$.

Here $T^{a}$ denotes the 4-velocity of the reference satellite and $X^{a}$ the connection vector pointing from the reference satellite to the second one. We then introduce the tetrad $\left\{\left(\mathbf{e}_{I}\right)^{a}, I=0,1,2,3\right\}$ carried by the reference satellite with $\left(\mathbf{e}_{0}\right)^{a}=T^{a}$. Through such local tetrad, we can map the above equation into the local frame of the reference satellite

$$
\begin{aligned}
\frac{d^{2}}{d \tau^{2}} X^{I}= & -2 \gamma_{J 0}^{I} \frac{d}{d \tau} X^{J}-\left(\frac{d}{d \tau} \gamma_{J 0}^{I}+\gamma^{K}{ }_{J 0} \gamma_{K 0}^{I}\right) X^{J} \\
& -K_{J}^{I} X^{J}
\end{aligned}
$$

Here, $\tau$ is the proper time measured by the reference satellite, and $\gamma_{J K}^{I}=\left(\mathbf{e}^{I}\right)^{v}\left(\mathbf{e}_{J}\right)^{\mu} \nabla_{\mu}\left(\mathbf{e}_{K}\right)_{\nu}$ are the Ricci rotation coefficients. According to the convention, the upper-latins $\{I, J, K, \ldots\}$ are used to index tensor components under the local tetrad $\left(\mathbf{e}_{I}\right)^{a}$. $\left(\mathbf{e}_{I}\right)^{\mu}$ can be viewed as the transformation matrix from local system to the Earth centered PN system, and $\left(\mathbf{e}^{I}\right)_{\mu}$ the inverse. The first lines of the right hand side of the above equation come from the gradients of inertial forces, which are resulted from the relative rotation of the local frame to the Fermi shifted frame. The last line comes from the tidal forces from spacetime curvature, where the the tidal matrix is defined as

$K_{J}^{I}=R_{\lambda \nu \rho}^{\mu} T^{\lambda} T^{\rho}\left(\mathbf{e}_{J}\right)^{v}\left(\mathbf{e}^{I}\right)_{\mu}$.

4.3 The order estimations of the geodesic deviations

Equation (13) is a system of ordinary differential equations that evaluated along the orbits of the reference satellite in its 
local frame. From dimensional analysis, up to the required 1PN level, Eq. (12) or (13) will have the following form

$\frac{d^{2} X}{d \tau^{2}} \sim \frac{1}{r} \frac{|X|}{r}\left(\mathscr{O}\left(\epsilon^{2}\right)+\mathscr{O}\left(\epsilon^{4}\right)+\cdots\right)$.

To clearly book-keep all the possible perturbation terms which appear in Eq. (13) and to understand the physical picture, we take the following approach. We first ignore the rotation of Earth, and the metric now reduce to the 1PN approximation of a spherical symmetric spacetime

$g_{\mu \nu}^{\mathbf{S}}=\left(\begin{array}{cccc}-1+\frac{2 M}{r}-\frac{2 \beta M^{2}}{r^{2}} & 0 & 0 & 0 \\ 0 & 1+\frac{2 \gamma M}{r} & 0 & 0 \\ 0 & 0 & 1+\frac{2 \gamma M}{r} & 0 \\ 0 & 0 & 0 & 1+\frac{2 \gamma M}{r}\end{array}\right)$.

For proof mass orbiting around the centered source in the above spherical symmetric spacetime, one can work out the equation of motion from the geodesic equation as [50]

$$
\begin{aligned}
\frac{d^{2} \mathbf{x}}{d t^{2}}= & -\frac{M}{r^{3}} \mathbf{x}+\frac{M}{r^{3}}\left(\left(\frac{2(\gamma+\beta) M}{r}-\gamma v^{2}\right) \mathbf{x}\right. \\
& +2(1+\gamma)(\mathbf{x} \cdot \mathbf{v}) \mathbf{v})+\frac{1}{r} \mathscr{O}\left(\epsilon^{6}\right) .
\end{aligned}
$$

At the 1PN level, circular orbits exist with the orbital frequency $\omega$

$\omega^{2}=\frac{1}{a^{2}}\left[\frac{M}{a}+(3-\gamma-2 \beta) \frac{M^{2}}{a^{2}}+\mathscr{O}\left(\epsilon^{6}\right)\right]$.

Therefore, for the ideal case, we set the two satellites to follow, one after another, the same circular orbit

$x^{1}=a \cos \Psi, x^{2}=a \cos i \sin \Psi, x^{3}=a \sin i \sin \Psi$,

where the longitude of ascending node $\Omega$ is set to be zero and $\Psi=\omega \tau$ denotes the orbital phase, see Fig. 1. Then, the most natural choice of the local tetrad for the evaluations of the range observable is the followings, that we set $\left(\mathbf{e}_{1}\right)^{a}$ along the direction of motion of the reference satellite, $\left(\mathbf{e}_{2}\right)^{a}$ along the radial direction, $\left(\mathbf{e}_{3}\right)^{a}=\left(\mathbf{e}_{1}\right)^{a} \times\left(\mathbf{e}_{2}\right)^{a}$ determined by the right hand rule and $\left(\mathbf{e}_{0}\right)^{a}=T^{a}$, see again Fig. 1 . Under such tetrad, the second satellite will stay static and the component of the connection vector will have the simple form $X^{I}=\left\{0, \rho_{0}, 0,0\right\}$, here $\rho_{0}$ measures the separation or the range between the two satellites. Such results in the local frame is due to the cancellations between the gradients of centripetal and centrifugal forces in this Earth pointing system.

Now, we "turn on" the Earth rotation and recover the $g_{0 i}$ components proportional to the source angular momentum
$J$, which can be taken as the 1PN perturbations act on the above circular orbits. The two satellites system now begins to "feel" the tidal force from the gravitomagnetic sector. Such gravitomagnetic tidal force will drive the second satellite to deviate from its original position relative to the reference one, which means that the components of the connection vector will now change with time

$X^{I}(\tau)=\left\{0, \rho_{0}+\delta^{1}(\tau), \delta^{2}(\tau), \delta^{3}(\tau)\right\}$.

Here, we need to estimate the magnitudes of this new family of small quantities, that the small deviations $\left\{\delta^{i}(\tau)\right\}$ driven by the gravitomagnetic tidal force, their time derivatives $\left\{\dot{\delta}^{i}(\tau)\right\}$ and the second time derivatives $\left\{\ddot{\delta}^{i}(\tau)\right\}$. From dimensional analysis, in the reference satellite local frame, the magnitude of the periodic gravitomagnetic force acting on the second satellite is

$\ddot{\delta^{i}} \sim \frac{J v \rho_{0}}{a^{4}} \sim \frac{1}{a} \frac{\rho_{0}}{a} \mathscr{O}\left(\epsilon^{4}\right)$,

and its frequency is of the orbital frequency $\omega \sim \sqrt{M / a^{3}}$. Thus one has the important order relations

$\dot{\delta}^{i} \sim \frac{J v \rho_{0}}{a^{4} \omega} \sim \frac{\rho_{0}}{a} \mathscr{O}\left(\epsilon^{3}\right)$,

$\delta^{i} \sim \frac{J v \rho_{0}}{a^{4} \omega^{2}} \sim \rho_{0} \mathscr{O}\left(\epsilon^{2}\right)$.

Therefore, with the above analysis and Eq. (15), up the to the 1PN level the geodesic deviation equation Eq. (13) will only contain the following terms

$\ddot{\delta}=\underbrace{\frac{1}{a} \frac{\rho_{0}}{a} \mathscr{O}\left(\epsilon^{2}\right)}_{\text {Newtonian part }}+\underbrace{\frac{1}{a} \dot{\delta} \mathscr{O}(\epsilon)+\frac{1}{a^{2}} \delta \mathscr{O}\left(\epsilon^{2}\right)+\frac{1}{a} \frac{\rho_{0}}{a} \mathscr{O}\left(\epsilon^{4}\right)}_{1 \text { PN part }}$.

At last, we work out the relation between the range variations $\delta \rho$ and the variations of the components of the connection vector. For nearly circular orbits, the relations between the components of the connection vector $X^{I}$ and the coordinates $x_{2}^{I}$ of the second satellite under the local frame is

$x_{2}^{I}=X^{I}+\mathscr{O}\left(\frac{|X|^{2}}{a}\right)$.

Then the relation between the variations of the coordinates of the second satellite $\delta x_{2}^{I}$ and the variations of the connection vector $\delta^{i}$ reads

$\delta x_{2}^{I}(\tau)=\delta^{I}(\tau)+a \frac{\rho_{0}^{2}}{a^{2}} \mathscr{O}\left(\epsilon^{2}\right)=\delta^{I}(\tau)\left(1+\mathscr{O}\left(\frac{\rho_{0}}{a}\right)+\cdots\right)$.

For GRACE and GRACE Follow On missions, the choice of $\rho_{0}$ ranges from 50 to $270 \mathrm{~km}$, and therefore $\frac{\rho_{0}}{a} \leq 3 \times 10^{-2}$. 
Thus, to the leading order, the range variation will have the simple from

$$
\begin{aligned}
\delta \rho(\tau)= & \delta^{1}(\tau)+\sum_{i=2,3} \mathscr{O}\left(\frac{\rho_{0}}{a}\right) \delta^{i}(\tau) \\
& +\left[\frac{\left(\delta^{2}(\tau)\right)^{2}}{2 \rho_{0}^{2}}+\frac{\left(\delta^{3}(\tau)\right)^{2}}{2 \rho_{0}^{2}}\right] \rho_{0} \\
= & \delta^{1}(\tau)+\sum_{i=2,3} \delta^{i}(\tau)\left[\mathscr{O}\left(\frac{\rho_{0}}{a}\right)+\mathscr{O}\left(\epsilon^{2}\right)\right] .
\end{aligned}
$$

\subsection{The equation of motion in the local frame}

As discussed in the last subsection, the geodesic deviation of the second satellite relative to the reference one along
For the tetrad attached to the reference satellite defined in the last subsection, we first set $\left(\mathbf{e}_{0}\right)^{a}=T^{a}$, and following the Gram-Schmidt process the three spacial bases can be worked out as

$$
\begin{aligned}
\left(\mathbf{e}_{1}\right)^{a}= & a \omega\left(\frac{\partial}{\partial t}\right)^{a}+\left(1+\frac{a^{2} \omega^{2}}{2}-\frac{\gamma M}{a}\right)\left[-\sin \Psi\left(\frac{\partial}{\partial x^{1}}\right)^{a}\right. \\
& \left.+\cos i \cos \Psi\left(\frac{\partial}{\partial x^{2}}\right)^{a}+\sin i \cos \Psi\left(\frac{\partial}{\partial x^{3}}\right)^{a}\right] \\
\left(\mathbf{e}_{2}\right)^{a}= & \left(1-\frac{\gamma M}{a}\right)\left[\cos \Psi\left(\frac{\partial}{\partial x^{1}}\right)^{a}+\cos i \sin \Psi\left(\frac{\partial}{\partial x^{2}}\right)^{a}\right. \\
& \left.+\sin i \sin \Psi\left(\frac{\partial}{\partial x^{3}}\right)^{a}\right], \\
\left(\mathbf{e}_{3}\right)^{a}= & \left(1-\frac{\gamma M}{a}\right)\left[\cos i\left(\frac{\partial}{\partial x^{3}}\right)^{a}-\sin i\left(\frac{\partial}{\partial x^{2}}\right)^{a}\right] .
\end{aligned}
$$

The transformation matrices then read

$\left(\mathbf{e}_{I}\right)^{\mu}=\left(\begin{array}{cccc}1+\frac{a^{2} \omega^{2}}{2}+\frac{M}{a} & -a \omega \sin \Psi & a \omega \cos i \cos \Psi & a \omega \sin i \cos \Psi \\ a \omega & -\left(1+\frac{a^{2} \omega^{2}}{2}-\frac{\gamma M}{a}\right) \sin \Psi & \left(1+\frac{a^{2} \omega^{2}}{2}-\frac{\gamma M}{a}\right) \cos i \cos \Psi & \left(1+\frac{a^{2} \omega^{2}}{2}-\frac{\gamma M}{a}\right) \sin i \sin \Psi \\ 0 & \left(1-\frac{\gamma M}{a}\right) \cos \Psi & \left(1-\frac{\gamma M}{a}\right) \cos i \sin \Psi & \left(1-\frac{\gamma M}{a}\right) \sin i \sin \Psi \\ 0 & 0 & -\left(1-\frac{\gamma M}{a}\right) \sin i & \left(1-\frac{\gamma M}{a}\right) \cos i\end{array}\right)$,

$\left(\mathbf{e}^{I}\right)_{\mu}=\left(\begin{array}{cccc}1+\frac{a^{2} \omega^{2}}{2}-\frac{M}{a} & -a \omega & 0 & 0 \\ a \omega \sin \Psi & -\left(1+\frac{a^{2} \omega^{2}}{2}+\frac{\gamma M}{a}\right) \sin \Psi & \left(1+\frac{\gamma M}{a}\right) \cos \Psi & 0 \\ -a \omega \cos i \cos \Psi & \left(1+\frac{a^{2} \omega^{2}}{2}+\frac{\gamma M}{a}\right) \cos i \cos \Psi & \left(1+\frac{\gamma M}{a}\right) \cos i \sin \Psi & -\left(1+\frac{\gamma M}{a}\right) \sin i \\ -a \omega \sin i \cos \Psi & \left(1+\frac{a^{2} \omega^{2}}{2}+\frac{\gamma M}{a}\right) \sin i \cos \Psi & \left(1+\frac{\gamma M}{a}\right) \sin i \sin \Psi & \left(1+\frac{\gamma M}{a}\right) \cos i\end{array}\right)$. the nearly circular orbit is produced by the 1PN gravitomagnetic tidal force, which are of terms proportional to $\frac{\rho_{0}}{a^{2}} \mathscr{O}\left(\frac{J v}{a^{4}}\right) \sim \frac{\rho_{0}}{a^{2}} \mathscr{O}\left(\epsilon^{4}\right)$. Therefore, from Eq. (23), to calculate such 1PN forces, one only need to work with the Newtonian (Keplerian) orbits. From the orbital choices of GRACE and GRACE FO missions, we work out here the range observable of non-dynamical CS gravity for the case of circular orbits, that Eq. (19). The effect of small orbital eccentricities $e \sim 2 \times 10^{-3}$ will be left in future works concerning real data analysis.

Along the circular orbit, Eq. (19), the 4-velocity of the reference satellite reads

$$
\begin{aligned}
T^{a}= & \frac{d t}{d \tau}\left(\frac{\partial}{\partial t}\right)^{a}+a \omega\left[-\sin \Psi\left(\frac{\partial}{\partial x^{1}}\right)^{a}\right. \\
& \left.+\cos i \cos \Psi\left(\frac{\partial}{\partial x^{2}}\right)^{a}+\sin i \cos \Psi\left(\frac{\partial}{\partial x^{3}}\right)^{a}\right]
\end{aligned}
$$

The ratio $\frac{d t}{d \tau}$ can be derived from the line element $d \tau^{2}=$ $-g_{\mu \nu} d x^{\mu} d x^{\nu}$ evaluated along the orbit

$$
\frac{d t}{d \tau}=1+\frac{a^{2} \omega^{2}}{2}+\frac{M}{a}+\mathscr{O}\left(\epsilon^{4}\right) .
$$

The explicit forms of the Christofell symbols $\Gamma_{\mu \nu}^{\lambda}$ and the tidal matrix from the Riemann curvature $K_{J}^{J}=R_{\lambda \nu \rho}^{\mu} T^{\lambda} T^{\rho}$ $\left(\mathbf{e}_{J}\right)^{\nu}\left(\mathbf{e}^{I}\right)_{\mu}$ are worked out up to $1 \mathrm{PN}$ level in Appendix A. Now, with all the results gathered here, we substitute the connection vector $X^{I}$, the transformation matrices Eqs. (27), (28), the Christoffel symbols Eqs. (B.1)-(B.6) and the curvature tidal matrices Eqs. (B.7)-(B.10) into the geodesic deviation equation Eq. (13). After the heavy works of simplifications and, according to Eq. (24), ignoring all the terms beyond $\frac{1}{a^{2}} \delta^{i} \mathscr{O}\left(\epsilon^{2}\right), \frac{1}{a} \dot{\delta}^{i} \mathscr{O}(\epsilon)$ and $\frac{\rho_{0}}{a^{2}} \mathscr{O}\left(\epsilon^{4}\right)$ we have the rather simple forms of the equations of motions that govern the deviations of the second satellite

$$
\begin{aligned}
& \ddot{\delta}^{1}(\tau)=-2 \omega \dot{\delta}^{2}(\tau)-\chi \frac{\rho_{0} J \omega \sin i \cos (\omega \tau)}{2 a^{3}}, \\
& \ddot{\delta}^{2}(\tau)=2 \omega \dot{\delta}^{1}(\tau)+3 \omega^{2} \delta^{2}(\tau)+\chi \frac{\rho_{0} J \omega \sin i \sin (\omega \tau)}{2 a^{3}} . \\
& \ddot{\delta}^{3}(\tau)=-\omega^{2} \delta^{3}(\tau)+2 \Delta \frac{\rho_{0} J \omega \sin i \cos (\omega \tau)}{a^{3}}
\end{aligned}
$$

As one should expect that the deviation $\delta^{3}(\tau)$ in the direction perpendicular to the $\left(\mathbf{e}_{1}\right)^{a}-\left(\mathbf{e}_{2}\right)^{a}$ plane will not couple into the first two equations. This is because the 1PN deviation $\delta^{3}(\tau)$ is perpendicular to both the along-track and 
radial directions, and can only alter the range $\rho(\tau)$ and the semi-major $a$ of the second satellite at the 2PN level. On the other hand, within the orbital plane, the deviation $\delta^{2}(\tau)$ along the radial direction does couple to the deviation $\delta^{1}(\tau)$ in the along-track direction and vise versa through the Coriolis effect.

An important feature of these geodesic deviation equations is that the 1PN deviations in the along-track and radial directions depend only on the tidal forces from the CS extension term in Eq. (2). The 1PN tidal forces proportional to the standard PN parameters $\left\{\gamma, \beta, \alpha_{1}, \alpha_{2}\right\}$ do not appear in the motions along these two directions. This is due to the fact that the gravitomagnetic gradients in standard parity-preserving metric theories evaluated along circular orbits will only affect the deviations in the transverse direction, which, as discussed above, will only affect the deviations within the orbital plane at $2 \mathrm{PN}$ level. Therefore, being a true advantage, the range variations at the orbital frequency can be used to distinguish CS gravity from standard parity-preserving metric theories (including GR).

At last, one should notice that the simple forms of the geodesics deviation equations under the local frame, that Eqs. (29)-(31), and the decoupling of the motions in the transverse direction from the along-track motions stay true only when the deviations are within the 1PN level, that $\delta^{i}(\tau)<\rho_{0} \mathscr{O}(\epsilon)$ and $\dot{\delta}^{i}(\tau)<\frac{\rho_{0}}{a} \mathscr{O}\left(\epsilon^{2}\right)$. When the deviations $\delta^{i}(\tau), \dot{\delta}^{i}(\tau)$ are beyond the 1PN level, terms of $\frac{1}{a^{2}} \delta^{i} \mathscr{O}\left(\epsilon^{4}\right)$ and $\frac{1}{a} \dot{\delta}^{i} \mathscr{O}\left(\epsilon^{3}\right)$ that are ignored at the first place will begin to play important roles in determining the relative motions. Also, the gravitomagnetic perturbations of the orbit of the reference satellite must be included into the geodesic deviation equations. Thus, for large deviations $\delta^{i} \geq \rho_{0} \mathscr{O}(\epsilon)$ and $\dot{\delta}^{i}(\tau) \geq \frac{\rho_{0}}{a} \mathscr{O}\left(\epsilon^{2}\right)$, Eqs. (29)-(31) will break down, and the full geodesic deviation equations will turn out to be very complicate and can hardly be solved analytically.

\subsection{The range observable}

The solutions of the equations of motions, that Eqs. (29) and (30), with general initial values $\left\{\delta_{0}^{1}, \delta_{0}^{2}, \dot{\delta}_{0}^{1}, \dot{\delta}_{0}^{2}\right\}$ are

$$
\begin{aligned}
\delta^{1}(\tau)= & -\frac{\chi \rho_{0} J \sin i \sin (\omega \tau)}{2 a^{3}} \tau-\frac{\chi \rho_{0} J \sin i(\cos (\omega \tau)-1)}{2 a^{3} \omega} \\
& +\delta_{0}^{1}-\frac{2 \dot{\delta}_{0}^{2}}{\omega}-3\left(\dot{\delta}_{0}^{1}+2 \dot{\delta}_{0}^{2} \omega\right) \tau+\frac{2 \dot{\delta}_{0}^{2} \cos (\omega \tau)}{\omega} \\
& +\frac{\left(4 \dot{\delta}_{0}^{1}+6 \delta_{0}^{2}\right) \sin (\omega \tau)}{\omega}, \\
\delta^{2}(\tau)= & \frac{\chi \rho_{0} J \sin i \cos (\omega \tau)}{4 a^{3}} \tau-\frac{\chi \rho_{0} J \sin i \sin (\omega \tau)}{4 a^{3} \omega} \\
& +\frac{2 \dot{\delta}_{0}^{1}}{\omega}+4 \delta_{0}^{2}+\frac{\dot{\delta}_{0}^{2} \sin (\omega \tau)}{\omega} \\
& -\frac{\left(2 \dot{\delta}_{0}^{1}+3 \delta_{0}^{2} \omega\right) \cos (\omega \tau)}{\omega} .
\end{aligned}
$$

The most interesting signal in the range variations is the growing terms in Eq. (32), which comes from the in-phase actions of the CS perturbations in the along-track and the radial directions. To be more specific, we write down the solutions with the ideal initial conditions $\delta_{0}^{1}=\delta_{0}^{2}=\dot{\delta}_{0}^{1}=$ $\dot{\delta}_{0}^{2}=0$ and recover the SI units

$$
\begin{aligned}
\delta \rho^{C S}(t)= & \delta^{1}(t)=-\frac{\chi G \rho_{0} J \sin i \sin (\omega t)}{2 c^{2} a^{3}} t \\
& -\frac{\chi G \rho_{0} J \sin i(\cos (\omega t)-1)}{2 c^{2} a^{3} \omega}, \\
\delta^{2}(t)= & \frac{\chi G \rho_{0} J \sin i \cos (\omega t)}{4 c^{2} a^{3}} t-\frac{\chi G \rho_{0} J \sin i \sin (\omega t)}{4 c^{2} a^{3} \omega} .
\end{aligned}
$$

Here we also replace the proper time $\tau$ of the reference satellite with the coordinate time of the PN coordinates system, since the difference between these two begins from $\mathscr{O}\left(\epsilon^{2}\right) t$ as showed in Eq. (26). In Figs. 2, 3 and 4, we illustrate the above solutions with the orbit options according to the GRACE and GRACE FO missions. The length units in these figures are chosen as $\chi \mathrm{m}$.

As discussed in the last subsection, the validities of the solutions Eqs. (32) and (33) are guaranteed under the conditions that the deviations between the two satellites should not exceed the 1PN level. Since the CS parameter $\chi$ for Earth orbit satellites was already constrained to be a rather small quantity $\chi \sim 0.17$ [21]. Therefore, it will take about $t \sim$ $\frac{a}{\chi c \mathscr{O}\left(\epsilon^{2}\right)} \sim 10^{7} y r s$ for these deviations to reach $\delta^{i} \sim \rho_{0} \mathscr{O}(\epsilon)$,
Fig. 2 The relative motions of the second satellite to the reference one in the $\left(\tilde{\mathbf{e}}_{1}\right)^{a}-\left(\tilde{\mathbf{e}}_{2}\right)^{a}$ plane. The orbits are chosen as near-circular polar orbits with altitudes as $460 / 250 \mathrm{~km}$. The satellites separations are chosen as $220 / 50 \mathrm{~km}$

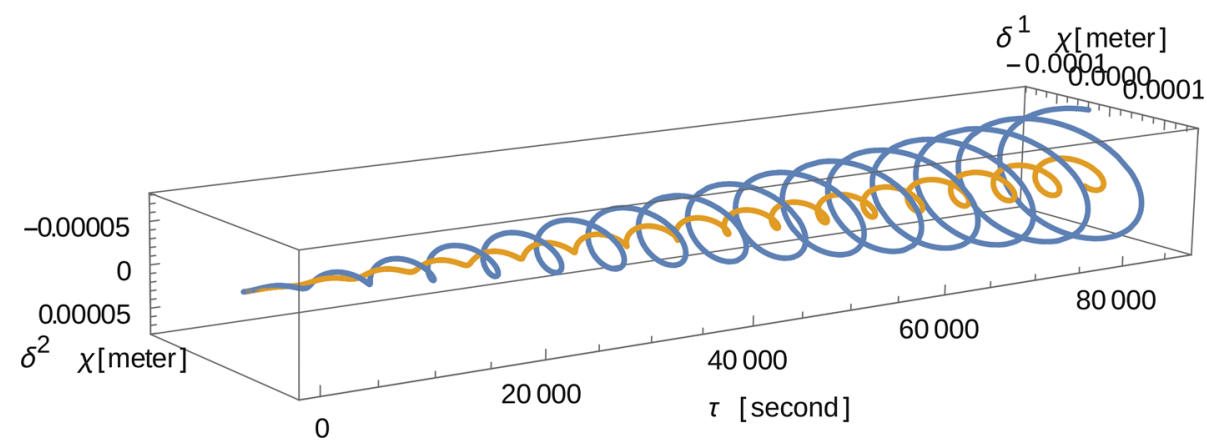


Fig. 3 The range variations in the along-track direction between the two satellites. The orbits are chosen as near-circular polar orbits with altitudes as $460 / 250 \mathrm{~km}$. The satellites separations are chosen as $220 / 50 \mathrm{~km}$

Fig. 4 The relative motions of the second satellite to the reference one in the radial direction. The orbits are chosen as near-circular polar orbits with altitudes as $460 / 250 \mathrm{~km}$. The satellites separations are chosen as $220 / 50 \mathrm{~km}$
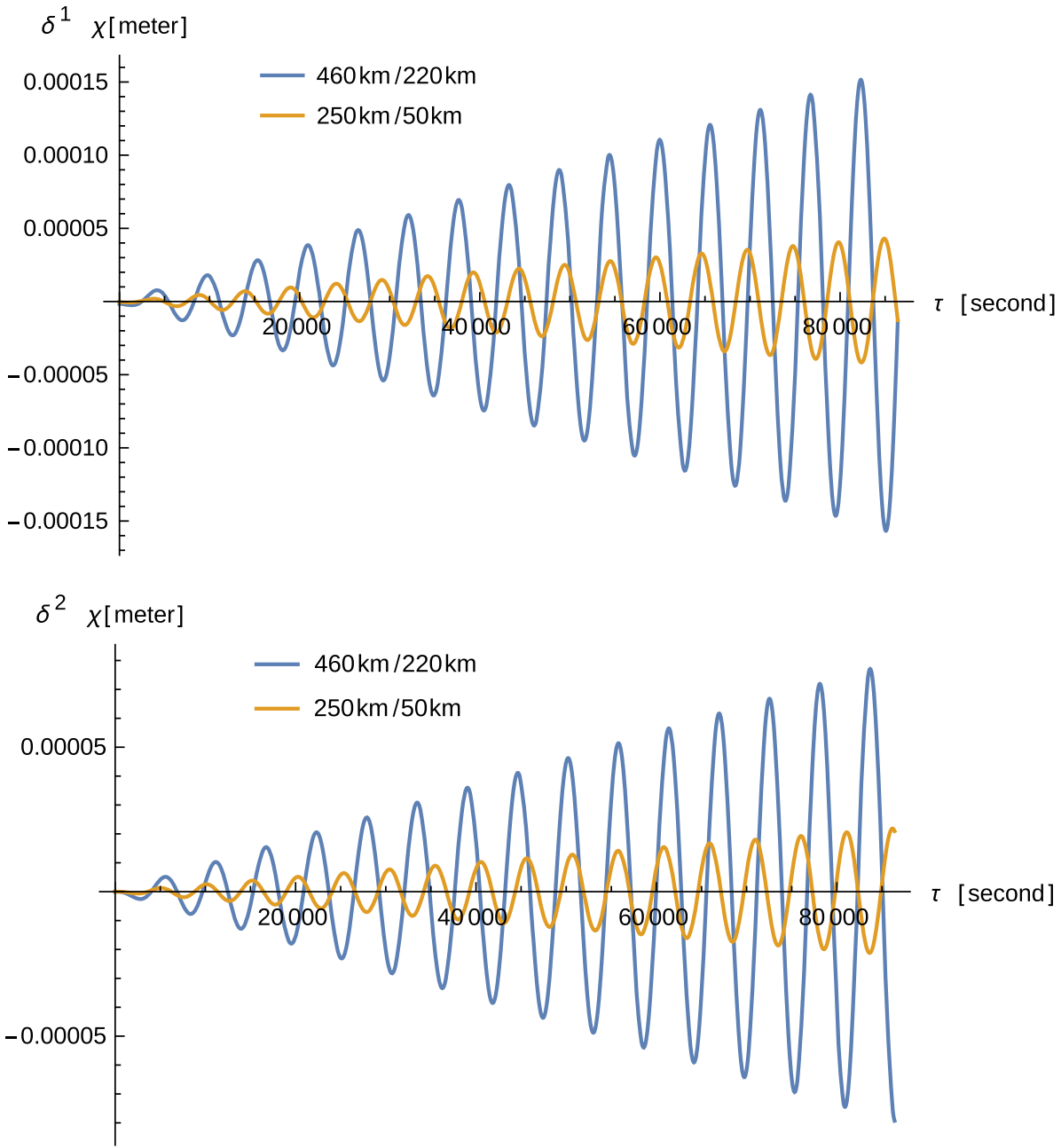

$\dot{\delta}^{i}(\tau) \sim \frac{\rho_{0}}{a} \mathscr{O}\left(\epsilon^{2}\right)$, and to break down the above solutions. For experiments that carried by the SST missions with at most 15 years life time, one needs not to worry about this issue.

\section{The estimations of the measurement accuracy and the concluding remarks}

From Eq. (34), the magnitude of the CS range signal $\left\|\delta \rho^{C S}(t)\right\|$ grows like $\frac{\chi G \rho_{0} J}{2 c^{2} a^{3}} t$. According to the orbits of the GRACE mission (see Table 1) and the possible orbit choices of the GRACE FO mission, we have $\left\|\delta \rho^{C S}(t)\right\| \sim$ $150 N \chi \mu \mathrm{m} /$ day for the $460 \mathrm{~km}$ altitude $220 \mathrm{~km}$ separation option and $\left\|\delta \rho^{C S}(t)\right\| \sim 40 N \chi \mu \mathrm{m} /$ day for the $250 \mathrm{~km}$ altitude $50 \mathrm{~km}$ separation option, here $N$ denotes the days of the free flight. GRACE generally need 2-4 orbits maneuvers per year, therefore the CS range signal accumulated in each free flight will reach to a few $\chi \mathrm{cm}$. As mentioned in Sect. 2, the accuracy of the range measurements near $10^{-4} \mathrm{~Hz}$ is about $1-2 \mathrm{~cm}$ for GRACE [42]. Therefore, with the proper data analysis methods, such as matched filtering and etc., the data from each free flight of GRACE (about $1.3 \times 10^{7} \mathrm{~s}$ ) can in principle set the constraint on the CS parameter as $\chi \leq 3.6 \times 10^{-4}$. From Eq. (8), and recovering the SI units, we have

$M_{C S}=\frac{4 \hbar c}{\chi a}$.

Therefore, the length scale and the mass scale of the nondynamical CS gravity to will be constrained as

$32 \pi \alpha \dot{\theta} \leq 2.5 \mathrm{~km}, \quad M_{C S} \geq 3.1 \times 10^{-10} \mathrm{eV}$.

The combination of the twelve years data of GRACE may improve the constraint to $M_{C S} \geq 1.9 \times 10^{-9} \mathrm{eV}$. For the future GRACE FO mission that re-flies the GRACE orbits, the accuracy of the range measurements around $10^{-4} \mathrm{~Hz}$ is about $100 \mu \mathrm{m}$ [45]. One then expects the constraint from the data of one free flight to be $\chi \leq 2.4 \times 10^{-6}$, which means that the length and mass scale of non-dynamical CS gravity can be constrained to

$32 \pi \alpha \dot{\theta} \leq 0.017 \mathrm{~km}, \quad M_{C S} \geq 4.6 \times 10^{-8} \mathrm{eV}$.

For the GRACE FO mission that flies the $220 \mathrm{~km}$ altitude $50 \mathrm{~km}$ separation option, the accuracy of the range mea- 
surements around $10^{-4} \mathrm{~Hz}$ is about $10 \mu \mathrm{m}$ [44]. Then the constraints from one free flight will be

$32 \pi \alpha \dot{\theta} \leq 6.0 \mathrm{~m}, \quad M_{C S} \geq 1.3 \times 10^{-7} \mathrm{eV}$.

The combination of the total nominal 5 years data of GRACE FO will further improve the constraints for about 3-4 times.

At last, we conclude this theoretical analysis with a brief discussions on the corresponding data analysis procedure and the future plans following this results. The SST missions such as GRACE and GRACE FO, designed originally for satellite geodesies, may provide us the strongest tests and constraints on the CS modified gravity up to now. While, the corresponding data analysis procedure will form an non-trivial task, since the frequency band around $10^{-4} \mathrm{~Hz}$ is affected by several noise sources, such the solar radiation pressure, Earth albedo, Earth atmosphere, attitude disturbances and etc.. One needs to employ the data from accelerometers, star sensors, magnetic torques and etc. to carefully remove the non-conservative forces subjected to the spacecrafts. The range signals produced by Earth gravity multiples generally lie in the frequency band much higher than the orbital frequency, and can then be removed by proper low pass filters. Take $J_{2}$ field for example, which is the strongest multiple component producing signal with the lowest frequency. To the leading order, the $J_{2}$ field will give rise to a range signal along the nearly circular orbits

$\delta \rho^{J_{2}}(t)=\frac{21 G C_{20} \rho_{0} R^{2} \sin ^{2} i \cos (2 \omega t)}{8 a^{2}}$,

which has twice the orbital frequency and can also be removed with proper low pass filters. To summarize, one has to start with the level $1 \mathrm{~b}$ data in searching for the CS signals, which forms a rather complicate task and will be left in future works.

Acknowledgments This work was supported by the NSFC Grands No. 11305255, No. 41104075 and Central Universities funds (CHD2009JC100 and 2014G3262010).

Open Access This article is distributed under the terms of the Creative Commons Attribution 4.0 International License (http://creativecomm ons.org/licenses/by/4.0/), which permits unrestricted use, distribution, and reproduction in any medium, provided you give appropriate credit to the original author(s) and the source, provide a link to the Creative Commons license, and indicate if changes were made.

Funded by SCOAP ${ }^{3}$.

\section{Appendix A: The standard PPN metric}

The standard PPN metric has the form [7]

$$
\begin{aligned}
g_{00}= & -1+2 U-2 \beta U^{2}-2 \xi \Phi_{W} \\
& +\left(2 \gamma+2+\alpha_{3}+\zeta_{1}-2 \xi\right) \Phi_{1} \\
& +2\left(3 \gamma-2 \beta+1+\zeta_{2}+\xi\right) \Phi_{2}+2\left(1+\zeta_{3}\right) \Phi_{3}
\end{aligned}
$$

$$
\begin{aligned}
& +2\left(3 \gamma+3 \zeta_{4}-2 \xi\right) \Phi_{4} \\
& -\left(\zeta_{1}-2 \xi\right) \mathscr{A}-\left(\alpha_{1}-\alpha_{2}-\alpha_{3}\right) w^{2} U-\alpha_{2} w^{i} w^{j} U_{i j} \\
& +\left(2 \alpha_{3}-\alpha_{1}\right) w^{i} V_{i}+\mathscr{O}\left(\epsilon^{6}\right), \\
g_{0 i}= & -\frac{1}{2}\left(4 \gamma+3+\alpha_{1}-\alpha_{2}+\zeta_{1}-2 \xi\right) V_{i} \\
& -\frac{1}{2}\left(1+\alpha_{2}-\zeta_{1}+2 \xi\right) W_{i} \\
& -\frac{1}{2}\left(\alpha_{1}-2 \alpha_{2}\right) w_{i} U-\alpha_{2} w^{j} U_{i j}+\mathscr{O}\left(\epsilon^{5}\right), \\
g_{i j}= & (1+2 \gamma U) \delta_{i j}+\mathscr{O}\left(\epsilon^{4}\right),
\end{aligned}
$$

where the PN potentials read

$$
\begin{aligned}
U & =\int \frac{\rho^{\prime}}{\left|\mathbf{x}-\mathbf{x}^{\prime}\right|} d^{3} x^{\prime}, \quad \Phi_{1}=\int \frac{\rho^{\prime} v^{\prime 2}}{\left|\mathbf{x}-\mathbf{x}^{\prime}\right|} d^{3} x^{\prime}, \\
\Phi_{2} & =\int \frac{\rho^{\prime} U^{\prime}}{\left|\mathbf{x}-\mathbf{x}^{\prime}\right|} d^{3} x^{\prime}, \quad \Phi_{3}=\int \frac{\rho^{\prime} \Pi^{\prime}}{\left|\mathbf{x}-\mathbf{x}^{\prime}\right|} d^{3} x^{\prime}, \\
\Phi_{4} & =\int \frac{p^{\prime}}{\left|\mathbf{x}-\mathbf{x}^{\prime}\right|} d^{3} x^{\prime}, \quad V_{i}=\int \frac{\rho^{\prime} v^{\prime}}{\left|\mathbf{x}-\mathbf{x}^{\prime}\right|} d^{3} x^{\prime}, \\
W_{i} & =\int \frac{\rho^{\prime}\left[\mathbf{v}^{\prime} \cdot\left(\mathbf{x}-\mathbf{x}^{\prime}\right)\right]\left(x^{i}-x^{\prime i}\right)}{\left|\mathbf{x}-\mathbf{x}^{\prime}\right|^{3}} d^{3} x^{\prime}, \\
U_{i j} & =\int \frac{\rho^{\prime}\left(x^{i}-x^{\prime i}\right)\left(x^{j}-x^{\prime j}\right)}{\left|\mathbf{x}-\mathbf{x}^{\prime}\right|^{3}} d^{3} x^{\prime}, \\
\mathscr{A} & =\int \frac{\rho^{\prime}\left[\mathbf{v}^{\prime} \cdot\left(\mathbf{x}-\mathbf{x}^{\prime}\right)\right]^{2}}{\left|\mathbf{x}-\mathbf{x}^{\prime}\right|^{3}} d^{3} x^{\prime}, \\
\Phi_{W} & =\int \frac{\rho^{\prime} \rho^{\prime \prime}\left(\mathbf{x}-\mathbf{x}^{\prime}\right)}{\left|\mathbf{x}-\mathbf{x}^{\prime}\right|^{3}} \cdot\left(\frac{\mathbf{x}^{\prime}-\mathbf{x}^{\prime \prime}}{\left|\mathbf{x}^{\prime}-\mathbf{x}^{\prime \prime}\right|}-\frac{\mathbf{x}-\mathbf{x}^{\prime \prime}}{\left|\mathbf{x}-\mathbf{x}^{\prime \prime}\right|}\right) d^{3} x^{\prime} d^{3} x^{\prime \prime} .
\end{aligned}
$$

The matter variables are the rest mass density $\rho$, pressure $p$, coordinate velocity of the matter field $v^{i}$, internal energy per unit mass $\Pi$ and the coordinate velocity of the PPN coordinate system relative to the mean rest-frame of the universe $w^{i}$. The PN orders read

$v \sim \mathscr{O}(\epsilon), \quad v^{2} \sim U \sim \Pi \sim \frac{p}{\rho} \sim \mathscr{O}\left(\epsilon^{2}\right)$.

The standard PN parameters $\left\{\gamma, \beta, \xi, \alpha_{1}, \alpha_{2}, \alpha_{3}, \zeta_{1}, \zeta_{2}\right.$, $\left.\zeta_{3}, \zeta_{4}\right\}$ have the following meanings. The parameters $\gamma$ and $\beta$ are the usual Eddington-Robertson-Schiff parameters used to describe the "classical" tests of GR and are in some sense the most important ones. For GR $\gamma=\beta=1$ are the only non-vanishing parameters. The parameter $\xi$ measures the preferred-location effects, $\left\{\alpha_{1}, \alpha_{2}, \alpha_{3}\right\}$ measure the preferred-frame effects and $\left\{\alpha_{3}, \zeta_{1}, \zeta_{2}, \zeta_{3}, \zeta_{4}\right\}$ measure the violations of global conservation laws for total momentum. The up-to-date values of these parameters are summarized in Table 2 [7].

\section{Appendix B: The Christoffel symbol and the tidal tensor}

The components of the Christoffel symbols $\Gamma_{\rho \lambda}^{\mu}$ under the PN coordinate system of Sect. 4 and the tidal matrix from the 
Riemann curvature $K_{J}{ }^{I}=R_{\lambda \nu \rho}{ }^{\mu} T^{\lambda} T^{\rho}\left(\mathbf{e}_{J}\right)^{\nu}\left(\mathbf{e}^{I}\right)_{\mu}$ along the orbit Eq. (19) are worked out as follows.

Here, we write down the complicate Christoffel symbols into matrix forms.

$\Gamma_{0 \mu}^{0}=\frac{M(r-2(\beta-1) M)}{r^{4}}\left(\begin{array}{c}0 \\ x^{1} \\ x^{2} \\ x^{3}\end{array}\right)$,

From Eqs. (14), (25), and (27)-(28), the tidal matrix $K_{J}^{I}=$ $K^{N}+K^{G E}+K^{G M}+K^{C S}$ along the circular orbit in the Earth pointing local frame can be worked out as

$$
K^{N}=\frac{M}{a^{3}}\left(\begin{array}{cccc}
0 & 0 & 0 & 0 \\
0 & 1 & 0 & 0 \\
0 & 0 & -2 & 0 \\
0 & 0 & 0 & 1
\end{array}\right),
$$

$K^{G E}=\frac{M}{a^{3}}\left(\begin{array}{cccc}0 & 0 & 0 & 0 \\ 0-\frac{(2 \beta+3 \gamma-2) M}{a} & 0 & 0 \\ 0 & 0 & \frac{(6 \beta+5 \gamma-5) M}{a}-(\gamma+2) a^{2} \omega^{2} & 0 \\ 0 & 0 & 0 & \frac{(-2 \beta-3 \gamma+2) M}{a}+(2 \gamma+1) \omega^{2} a^{2}\end{array}\right)$,

$$
K^{G M}=\frac{J \omega}{a^{3}}\left(\begin{array}{cccc}
0 & 0 & 0 & 0 \\
0 & 0 & 0 & -\frac{3}{2} \Delta \sin i \cos \Psi \\
0 & 0 & 3 \Delta \cos i & \frac{9}{2} \Delta \sin i \sin \Psi \\
0-\frac{3}{2} \Delta \sin i \cos \Psi & \frac{9}{2} \Delta \sin i \sin \Psi & -3 \Delta \cos i
\end{array}\right),
$$

$$
\begin{aligned}
\Gamma_{i j}^{1} & =-\frac{\gamma M}{r^{3}}\left(\begin{array}{ccc}
x^{1} & x^{2} & x^{3} \\
x^{2} & -x^{1} & 0 \\
x^{3} & 0 & -x^{1}
\end{array}\right), \\
\Gamma_{i j}^{2} & =-\frac{\gamma M}{r^{3}}\left(\begin{array}{ccc}
-x^{2} & x^{1} & 0 \\
x^{1} & x^{2} & x^{3} \\
0 & x^{3} & -x^{2}
\end{array}\right), \\
\Gamma_{i j}^{3} & =-\frac{\gamma M}{r^{3}}\left(\begin{array}{ccc}
-x^{3} & 0 & x^{1} \\
0 & -x^{3} & x^{2} \\
x^{1} & x^{2} & x^{3}
\end{array}\right),
\end{aligned}
$$

$$
K^{C S}=\frac{J \omega}{a^{3}}\left(\begin{array}{cccc}
0 & 0 & 0 & 0 \\
0 & 0 & -\frac{1}{4} \chi \sin i \sin \Psi & -\frac{1}{4} \chi \cos i \\
0-\frac{1}{4} \chi \sin i \sin \Psi & -\frac{3}{2} \chi \sin i \cos \Psi & 0 \\
0 & -\frac{1}{4} \chi \cos i & 0 & \frac{1}{2} \chi \sin i \cos \Psi
\end{array}\right) .
$$

Here, $K^{N}, K^{G E}, K^{G M}$ and $K^{C S}$ denote the gravitational tidal matrices from the Newtonian force, the 1PN gravitoelectric force, the gravitomagnetic force and the contributions from the CS modification.

$$
\Gamma_{i j}^{0}=\left(\begin{array}{c}
\frac{3 \Delta J\left(x^{2}\left(x^{1}\right)^{3}+x^{2}\left(\left(x^{2}\right)^{2}+\left(x^{3}\right)^{2}\right) x^{1}\right)}{+\frac{3 \chi J\left(3 x^{3}\left(x^{1}\right)^{2}-x^{7}\left(\left(x^{2}\right)^{2}+\left(x^{3}\right)^{2}\right)\right)}{2 r^{6}}} \\
+\frac{3 \Delta J\left(-\left(x^{3}\right)^{2}\left(x^{1}\right)^{2}-\left(x^{1}\right)^{4}+\left(x^{2}\right)^{2}\left(\left(x^{2}\right)^{2}+\left(x^{3}\right)^{2}\right)\right)}{2 x^{2} x^{3} x^{1}} \\
r^{6}
\end{array}\right.
$$

$$
\begin{array}{cc}
\frac{3 \Delta J\left(-\left(x^{3}\right)^{2}\left(x^{1}\right)^{2}-\left(x^{1}\right)^{4}+\left(x^{2}\right)^{2}\left(\left(x^{2}\right)^{2}+\left(x^{3}\right)^{2}\right)\right)}{2 r^{7}} & \frac{3 \Delta J\left(x^{2} x^{3}\left(x^{1}\right)^{2}+x^{2} x^{3}\left(\left(x^{2}\right)^{2}+\left(x^{3}\right)^{2}\right)\right)}{2 r^{7}} \\
+\frac{6 x^{2} x^{3} x^{1}}{r^{6}} & -\frac{\chi J\left(5\left(x^{1}\right)^{3}+\left(5\left(x^{2}\right)^{2}-19\left(x^{3}\right)^{2}\right) x^{1}\right)}{4 r^{6}} \\
-\frac{3 \Delta J\left(x^{2}\left(x^{1}\right)^{3}+x^{2}\left(\left(x^{2}\right)^{2}+\left(x^{3}\right)^{2}\right) x^{1}\right)}{r^{7}} & -\frac{3 \Delta J\left(x^{3}\left(x^{1}\right)^{3}+x^{3}\left(\left(x^{2}\right)^{2}+\left(x^{3}\right)^{2}\right) x^{1}\right)}{2 r^{7}} \\
-\frac{3 \chi J\left(x^{3}\left(x^{1}\right)^{2}+x^{3}\left(\left(x^{3}\right)^{2}-3\left(x^{2}\right)^{2}\right)\right)}{2 r^{6}} & -\frac{\left.\chi J\left(5 x^{2}\left(x^{1}\right)^{2}+x^{2}\left(5 x^{2}\right)^{2}-19\left(x^{3}\right)^{2}\right)\right)}{4 r^{6}} \\
-\frac{3 \Delta J\left(x^{3}\left(x^{1}\right)^{3}+x^{3}\left(\left(x^{2}\right)^{2}+\left(x^{3}\right)^{2}\right) x^{1}\right)}{2 r^{7}} & \frac{2 \chi J x^{3}\left(-2\left(x^{1}\right)^{2}-2\left(x^{2}\right)^{2}+\left(x^{3}\right)^{2}\right)}{r^{6}}
\end{array}
$$




\section{References}

1. R.H. Dicke, Relativity, Groups and Topology (Gordon and Breach, New York, 1964)

2. K. Nordtvedt, Phys. Rev. 169, 1014 (1968). doi:10.1103/PhysRev. 169.1014

3. C.M. Will, Astrophys. J. 163, 611 (1971). doi:10.1086/150804

4. C.M. Will, J.K. Nordtvedt, Astrophys. J. 177, 757 (1972)

5. C.M. Will, Astrophys. J. 185, 31 (1973). doi:10.1086/152394

6. S.G. Turyshev, Ann. Rev. Nucl. Part. Sci. 58, 207 (2008). doi:10. 1146/annurev.nucl.58.020807.111839

7. C.M. Will, Liv. Rev. Relat. 17(4) (2014). doi:10.12942/lrr-2014-4

8. J.F. Navarro, C.S. Frenk, S.D.M. White, Astrophys. J. 462, 563 (1996). doi:10.1086/177173

9. P.J.E. Peebles, B. Ratra, Rev. Mod. Phys. 75, 559 (2003). doi:10. 1103/RevModPhys.75.559

10. G. Bertone, D. Hooper, J. Silk, Phys. Rep. 405, 279 (2005). doi:10. 1016/j.physrep.2004.08.031

11. D. Clowe, M. Bradač, A.H. Gonzalez, M. Markevitch, S.W. Randall, C. Jones, D. Zaritsky, Astrophys. J. Lett. 648, L109 (2006). doi:10.1086/508162

12. M. Li, X.D. Li, S. Wang, Y. Wang, Front. Phys. 8, 828 (2013). doi:10.1007/s11467-013-0300-5

13. T. Clifton, P.G. Ferreira, A. Padilla, C. Skordis, Phys. Rep. 513, 1 (2012). doi:10.1016/j.physrep.2012.01.001

14. M. Niedermaier, M. Reuter, Liv. Rev. Relat. 9, 5 (2006). doi:10. 12942/lrr-2006-5

15. S. Deser, R. Jackiw, S. Templeton, Ann. Phys. 140, 372 (1982)

16. B.A. Campbell, M.J. Duncan, N. Kaloper, K.A. Olive, Phys. Lett. B 251, 34 (1990). doi:10.1016/0370-2693(90)90227-W

17. B.A. Campbell, M.J. Duncan, N. Kaloper, K.A. Olive, Nucl. Phys. B 351, 778 (1991). doi:10.1016/S0550-3213(05)80045-8

18. R. Jackiw, S.Y. Pi, Phys. Rev. D 68, 104012 (2003). doi:10.1103/ PhysRevD.68.104012

19. S. Alexander, N. Yunes, Phys. Rep. 480, 1 (2009). doi:10.1016/j. physrep.2009.07.002

20. J. Polchinski, in String Theory: Volume 2, Superstring Theory and Beyond. Cambridge Monographs on Mathematical Physics (Cambridge University Press, Cambridge, 1998). http://books.google. com.hk/books?id=WKatSc5pjOgC

21. T.L. Smith, A.L. Erickcek, R.R. Caldwell, M. Kamionkowski, Phys. Rev. D 77, 024015 (2008). doi:10.1103/PhysRevD.77. 024015

22. I. Ciufolini, E. Pavlis, Nature 431, 958 (2004). doi:10.1038/ nature 03007

23. I. Ciufolini, Nature 449, 41 (2007). doi:10.1038/nature06071

24. I. Ciufolini, E.C. Pavlis, J. Ries, R. Koenig, G. Sindoni, A. Paolozzi, H. Newmayer, in Astrophysics and Space Science Library, Astrophysics and Space Science Library, vol. 367, ed. by I. Ciufolini, R.A.A. Matzner (2010), p. 371. doi:10.1007/978-90-481-3735-0_ 17

25. C.W.F. Everitt, D.B. Debra, B.W. Parkinson, J.P. Turneaure, J.W. Conklin, M.I. Heifetz, G.M. Keiser, A.S. Silbergleit, T. Holmes, J. Kolodziejczak, M. Al-Meshari, J.C. Mester, B. Muhlfelder, V.G. Solomonik, K. Stahl, P.W. Worden Jr, W. Bencze, S. Buchman, B. Clarke, A. Al-Jadaan, H. Al-Jibreen, J. Li, J.A. Lipa, J.M. Lockhart, B. Al-Suwaidan, M. Taber, S. Wang, Phys. Rev. Lett. 106(22), 221101 (2011). doi:10.1103/PhysRevLett.106.221101
26. K. Konno, T. Matsuyama, Y. Asano, S. Tanda, Phys. Rev. D 78, 024037 (2008). doi:10.1103/PhysRevD.78.024037

27. N. Yunes, D.N. Spergel, Phys. Rev. D 80, 042004 (2009). doi:10. 1103/PhysRevD.80.042004

28. Y. Ali-Haimoud, Phys. Rev. D 83, 124050 (2011). doi:10.1103/ PhysRevD.83.124050

29. N. Yunes, F. Pretorius, Phys. Rev. D 79, 084043 (2009). doi:10. 1103/PhysRevD.79.084043

30. Y. Ali-Haimoud, Y. Chen, Phys. Rev. D 84, 124033 (2011). doi:10. 1103/PhysRevD.84.124033

31. K. Yagi, N. Yunes, T. Tanaka, Phys. Rev. D 86, 044037 (2012). doi:10.1103/PhysRevD.86.044037

32. S. Chen, J. Jing, Class. Q. Grav. 27, 225006 (2010). doi:10.1088/ 0264-9381/27/22/225006

33. K. Yagi, L.C. Stein, N. Yunes, T. Tanaka, Phys. Rev. D 87, 084058 (2013). doi:10.1103/PhysRevD.87.084058

34. F. Vincent, Class. Q. Grav. 31, 025010 (2013). doi:10.1088/ 0264-9381/31/2/025010

35. C.F. Sopuerta, N. Yunes, Phys. Rev. D 80, 064006 (2009). doi:10. 1103/PhysRevD.80.064006

36. D. Garfinkle, F. Pretorius, N. Yunes, Phys. Rev. D 82, 041501 (2010). doi:10.1103/PhysRevD.82.041501

37. P. Pani, V. Cardoso, L. Gualtieri, Phys. Rev. D 83, 104048 (2011). doi:10.1103/PhysRevD.83.104048

38. P. Canizares, J.R. Gair, C.F. Sopuerta, Phys. Rev. D 86, 044010 (2012). doi:10.1103/PhysRevD.86.044010

39. S. Alexander, N. Yunes, Phys. Rev. Lett. 99, 241101 (2007). doi:10. 1103/PhysRevLett.99.241101

40. S. Alexander, N. Yunes, Phys. Rev. D 75, 124022 (2007). doi:10. 1103/PhysRevD.75.124022

41. B.D. Tapley, S. Bettadpur, M. Watkins, C. Reigber, Geophys. Res. Lett. 31, L09607 (2004). doi:10.1029/2004GL019920

42. J. Kim, P. Roesset, S. Bettadpur, B. Tapley, M. Watkins, in Gravity, Geoid and Geodynamics 2000, International Association of Geodesy Symposia, vol. 123, ed. by M. Sideris (Springer, Berlin, Heidelberg, 2002), pp. 103-108. doi:10.1007/ 978-3-662-04827-6_17

43. R. Pierce, J. Leitch, M. Stephens, P. Bender, R. Nerem, Appl. Opt. 47, 5007 (2008). doi:10.1364/AO.47.005007

44. B.D. Loomis, R.S. Nerem, S.B. Luthcke, J. Geod. 86, 319 (2012). doi:10.1007/s00190-011-0521-8

45. B.S. Sheard, G. Heinzel, K. Danzmann, D.A. Shaddock, W.M. Klipstein, W.M. Folkner, J. Geod. 86, 1083-1095 (2012). doi:10. 1007/s00190-012-0566-3

46. L. Iorio, Adv. Space Res. 50, 334 (2012)

47. L. Iorio, Gen. Relat. Gravit. 44, 1753 (2012). doi:10.1007/ s10714-012-1365-0

48. L. Iorio, Annalen der Physik 524, 371 (2012). doi:10.1002/andp. 201200060

49. C. Will, Theory and Experiment in Gravitational Physics (Cambridge University Press, Cambridge, 1993)

50. G. Petit, B. Luzum et al., IERS Tech. Note 36, 1 (2010) 